\title{
Actores sociales y sustentabilidad ambiental. Un acercamiento a las organizaciones que influyen en la gestión de los recursos naturales en Marismas Nacionales Sinaloa
}

\author{
Joel Bojórquez Sauceda* \\ Gloria Jovita Guadarrama Sánchez**
}

Recepción: 28 de enero de 2017 Aceptación: 8 de agosto de 2017

Resumen Actualmente la consideración de la sustentabilidad en la gestión de los recursos naturales se ha vuelto un asunto social de suma importancia. El propósito de este estudio es identificar y caracterizar a los actores sociales que participan en la gestión de los recursos del sistema socioecológico Marismas Nacionales Sinaloa, con base en el enfoque de gobernanza ambiental. La caracterización se sustenta en investigación hemerográfica y se respalda con una encuesta a integrantes de las organizaciones identificadas como actores sociales. Los resultados ubican a las organizaciones públicas y sociales relacionadas con la conservación y el desarrollo de los recursos naturales en la zona de estudio.

PALABRAS CLAVE: actores sociales, gestión, Marismas Nacionales Sinaloa, recursos naturales, sistemas socioecológicos.

Abstract At present, consideration of sustainability in the management of natural resources has become a very important social issue. The purpose of this study is to identify and characterize the social actors involved in the management of the resources of the socio-ecological system Marismas Nacionales Sinaloa, based on the environmental governance approach. Characterization is based on hemerographic research and also supported by a survey of members of organizations identified as social actors. The results locate the public and social organizations that have to do with the use, conservation and development of natural resources in the study area.

KEY WORDS: social actors, management, Marismas Nacionales Sinaloa, natural resources, socioecological systems.

\footnotetext{
* Profesor-investigador de la Universidad Autónoma de Sinaloa, rockyboj@hotmail.com

** Profesora-investigadora en El Colegio Mexiquense, A.C., ggloriags@prodigy.net.mx
} 


\section{Introducción}

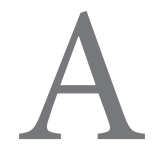

partir de la década de los ochenta del siglo XX se reconoce que la conservación de los recursos naturales y la sustentabilidad de ellos son fundamentales para el desarrollo nacional y para mejorar las condiciones de bienestar de sus habitantes; por eso se torna necesario revisar las acciones públicas que hacen posible atender los problemas que presenta la gestión de los recursos naturales para alcanzar mejores resultados con respecto a la sustentabilidad de los mismos. Asimismo, resulta evidente que los avances tienen que ver con la gestión pública como un proceso en que los actores que intervienen son relevantes en el uso, aprovechamiento y regulación de los recursos, y que el papel que desempeñan es fundamental en el control y la gobernanza de los bienes colectivos.

El Programa de las Naciones Unidas para el Medio Ambiente, (PNUMA, 2009, p. 2) establece que la gobernanza ambiental abarca las reglamentaciones, prácticas, políticas e instituciones que configuran la manera en que las personas interactúan con el medio ambiente. En la buena gobernanza ambiental se tienen en cuenta las funciones de todos los agentes que en él repercuten. Desde los gobiernos hasta las organizaciones no gubernamentales (ONG), el sector privado y la sociedad civil, la cooperación es fundamental para lograr una gobernanza eficaz que nos pueda ayudar a transitar hacia un futuro más sostenible.

Aunque tradicionalmente la gobernanza ambiental ha sido el dominio del Estado, la frecuente falta de éxito ha cuestionado su papel predominante en estos procesos y su capacidad para alcanzar las metas de conservación (Ostrom, 2007, p. 184). Esto ha obligado a la organización e intervención de la sociedad civil en los procesos de gobernanza, lo que ha tenido como resultado una más amplia gama de arreglos institucionales y legislativos en materia de gobernanza ambiental a escala local, regional, nacional e internacional.

Los actores sociales son los depositarios y activos de la gestión de los recursos naturales. A través de ellos se realizan las acciones que tienen como resultado su equilibrio o la sobreexplotación o degradación, además de que representan los intereses económicos, políticos y sociales que se involucran en su manejo. El estudio que aquí se presenta hace una exploración de los actores que influyen en el manejo de los recursos naturales en el sistema socioecológico de Marismas Nacionales Sinaloa (MNS), asumiendo que las organizaciones que los integran son los actores principales. Para ese propósito, a partir de una base de datos hemerográfica, las organizaciones fueron clasificadas de acuerdo con su papel y posición en la gestión de los recursos naturales, y se aplicó una encuesta para conocer las características de cada organización, sus intereses, formas de ocupación del espacio y su relación con otros actores. 
El ejercicio de caracterización ofrece un acercamiento contextual de la conformación social del territorio, identifica y ubica actores, entrega información para la toma de decisiones y contribuye a la preparación, programación y ejecución de las políticas públicas para la gestión de los recursos naturales. En el área de MNS el conocimiento de los actores sociales involucrados en la gestión de los recursos naturales presenta insuficiencias sustanciales con respecto a su cuantificación y sus características, así como las relaciones entre ellos, con el propósito de influir en el proceso de manejo, por lo que actualizar el conocimiento sobre su intervención en los procesos de sustentabilidad es importante para las estrategias de planeación, toma de decisiones e implementación de políticas públicas dirigidas a la gestión de recursos naturales.

En ese marco, el análisis está orientado a subsanar esta deficiencia mediante la identificación y caracterización de las organizaciones involucradas en el manejo de los recursos naturales en esta zona. Una consideración preliminar que sirve de guía a la exploración es que la gestión sectorizada de los recursos por las organizaciones y el papel que asumen en las interacciones con otros actores responde a intereses particulares de cada uno de los sectores, lo cual se traduce en obstáculos para el manejo sustentable de los recursos.

Con esos elementos, la exploración propone identificar y caracterizar las organizaciones que influyen en el manejo de los recursos naturales en la zona de Marismas Nacionales Sinaloa, ubicar su participación en las actividades socioeconómicas sustentadas en recursos naturales y clasificarlas de acuerdo con su papel en la gestión, su escala de influencia y su jurisdicción. La identificación está basada en la revisión de la base de datos hemerográficos del Sistema Integrado de Información de los Recursos Naturales (SIIRENA) de la Facultad de Ciencias del Mar (FACIMAR) de la Universidad Autónoma de Sinaloa (UAS), utilizando como herramienta el paquete informático para el análisis de datos cualitativos Atlas ti. 6.2, y sigue la metodología propuesta por el Instituto de Investigación de Recursos Biológicos Alexander von Humboldt (IAVH) y el Centro de Cooperación Indígena (CECOÍN) de Colombia (2005). La tipología para la identificación de actores relevantes para el manejo está basada en la Guía de identificación de actores clave de la Comisión Nacional del Agua (CNA) (2007) y en la tipología propuesta por Grimble y Wellard (1996) desde el enfoque de la gobernanza ambiental, la cual identifica tres tipos de actores en la gestión de recursos naturales, los enfocados en el uso de recursos (apropiadores), aquellos enfocados en el desarrollo (reguladores) y los enfocados en la conservación (orientadores). Cada tipo persigue objetivos divergentes y busca establecer un régimen particular de uso de los recursos en un territorio determinado.

Para establecer los rasgos y ubicar a los actores se aplicó una encuesta a una muestra representativa de las organizaciones que intervienen en la gestión de los 
recursos, utilizando la técnica de bola de nieve. Los actores fueron divididos previamente de acuerdo con su papel como apropiadores, reguladores y orientadores. La estructura de la encuesta para los apropiadores está orientada a obtener información relativa, entre otros temas, de sus actividades y métodos de producción, canales de comercialización, interacciones con el sistema socioecológico, programas de apoyo o subsidios a los que tienen acceso, posesión de derechos de acceso a los recursos, trámites y las restricciones que tiene su actividad y las interacciones con otros actores que influyen en el manejo de los recursos naturales.

Por otro lado, la estructura de la encuesta para las organizaciones gubernamentales está encaminada a obtener información referente al tipo de programas de apoyo o subsidios en operación, actividades de interés prioritario, tipo de actores que apoyan y objetivos de los apoyos, tipos de sanciones que aplican, los recursos regulados, el presupuesto que manejan las dependencias y en qué rubros lo aplican.

Para obtener información de los orientadores, la encuesta indaga principalmente sobre los programas que implementan, tipos de organizaciones que apoyan, el objetivo y las condiciones para otorgar apoyos. Además, inquiere acerca de las formas de fomentar el conocimiento y uso de las leyes o normas que reglamentan las actividades que gestionan, si están implicados en programas de inspección y vigilancia, la coordinación con otras organizaciones y cuáles son los recursos que les interesan.

Con base en las categorías arriba descritas, fueron construidas diferentes matrices que sustentan el análisis cualitativo y los resultados que se presentan. El papel en el manejo fue definido como la función que desempeñan las organizaciones en una zona o territorio, ya que ellas tienen objetivos diferentes. Algunas están dedicadas a la extracción de los recursos naturales, su uso o tanto este como la extracción; otras desempeñan el papel de regular, a través de marcos jurídicos, las actividades socioeconómicas, con lo cual inciden en el uso y aprovechamiento de los recursos, y por último, otras se dedican a orientar a otras organizaciones (productores y gubernamentales) en temas como aprovechamiento sustentable y gestión empresarial. Estos criterios permiten separar a las organizaciones de acuerdo con su papel en el manejo y establecer tres categorías analíticas para identificarlas: organizaciones de apropiadores, organizaciones de reguladores y organizaciones de orientadores.

El texto está estructurado en cuatro apartados. En el primero de ellos se abordan las perspectivas teóricas que dan cuenta de los conceptos utilizados como marco guía de la investigación. Un segundo apartado describe el área de estudio, asumiendo que es un sistema socioecológico, por lo que aborda la Región Marismas Nacionales Sinaloa desde su contexto natural, socioeconómico y jurídicoadministrativo. Un tercer apartado muestra los principales hallazgos del análisis. 
Finalmente, en el cuarto apartado se establece una serie de conclusiones a partir de los resultados más importantes.

\section{Las perspectivas sobre los actores sociales en la sustentabilidad ambiental}

\section{Marismas Nacionales Sinaloa como un sistema socioecológico}

Los sistemas litorales, como el de Marismas Nacionales Sinaloa, son sistemas abiertos, complejos en su estructura, estrechamente interrelacionados entre sí; tienen un funcionamiento y un carácter extremadamente dinámico, no siempre son fáciles de comprender y es difícil prever las repercusiones que tiene en ellos cualquier intervención humana. Los procesos que se registran son de diversa naturaleza: geodinámicos, hidrodinámicos, ecodinámicos, sociológicos o de mentalidad colectiva, productivos, administrativos, etc. (Barragán, 2004, p. 23).

Farhad (2012) conceptualiza las áreas litorales como sistemas socioecológicos ya que esta noción aporta un concepto "holístico, sistémico e integrador", que coloca al ser humano en la naturaleza. Por tanto, se entiende como un sistema complejo adaptativo en el que interactúan distintos componentes culturales, políticos, sociales, económicos, ecológicos, tecnológicos, etc. Esto implica que el enfoque de la gestión de los ecosistemas y recursos naturales no está centrado en los componentes del sistema sino en sus relaciones, interacciones y retroalimentaciones (Resilience Alliance, 2010).

Barragán (2003) divide el sistema costero en tres subsistemas: subsistema físico y natural, subsistema social y económico, y subsistema jurídico. Según Barragán (2004), pocos ámbitos geográficos y recursos registran intensidades de usos tan complejos y elevados, y con una tendencia claramente creciente, como la zona costera. Aquí converge un número inusual de usos y actividades que explican la gran concentración de asentamientos humanos, equipamientos e infraestructuras en ellos. Además:

...desde el punto de vista jurídico y administrativo, es preciso destacar una serie de aspectos, en algunos casos absolutamente singulares: a) la naturaleza pública de la mayor parte de las áreas marítimo-terrestres y marinas; b) el carácter público de los recursos vivos (peces, crustáceos, aves...) y no vivos (arena, petróleo, gas, aguas marinas...) de las mismas; c) la inusual convergencia de administraciones en la gestión de los intereses y asuntos públicos, tanto en lo referido a las escalas territoriales como a los sectores de Administración; d) el elevado número de intereses privados diferentes, 
que no siempre pueden convivir de forma armónica; e) la diversidad de los mecanismos e instrumentos de gestión establecidos; f) la dificultad para encontrar fórmulas que hayan tenido éxito en la ordenación del espacio y los recursos litorales (Barragán, 2004).

Ramírez Zavala, Ramírez Zavala, Bojórquez Sauceda, Santos Guzmán y Herrera y Cairo Lizárraga (2012) analizan la problemática ambiental de Marismas Nacionales Sinaloa estableciendo el contexto socioambiental de la región. Revelan la generación de una renovada inquietud de grupos ciudadanos en torno al proceso de desarrollo regional, lo que transforma la problemática observada en la zona en un asunto con creciente participación de los actores locales involucrados.

\section{Las organizaciones como actores sociales}

Con respecto al papel que desempeñan las organizaciones como actores sociales clave en la gestión de los recursos naturales, la Conagua (2007, p. 4) considera la identificación de ellos como "una herramienta que permite a los responsables de un proyecto, la recopilación sistemática, ordenada y actualizada de información estratégica, identificando y valorando aquellos actores que pueden colaborar y a los que hay que tomar en cuenta para evitar conflictos en el desarrollo de proyectos".

En esta exploración del papel del actor social se asume que puede ser un individuo o un grupo, organización, entidad, corporativo u organismo del sector público, social o privado, organización de la sociedad civil o agencia internacional que tenga relación directa o indirecta con la gestión de los recursos naturales. Las organizaciones consideradas aquí como actores sociales son entendidas como un "Conjunto de personas con intereses homogéneos que participan en un proyecto o propuesta. Son pieza principal de la relación entre sociedad y territorio. Pueden ser personas físicas o morales, grupos, organizaciones, redes, entidades gubernamentales o no gubernamentales y organismos internacionales que tienen una posición y participan directa o indirectamente" (Conagua, 2007, p. 24).

El Banco Mundial (2007) precisa que los actores sociales pueden ser comunidades o personas afectadas a nivel local y sus representantes oficiales y extraoficiales, autoridades de gobierno a nivel nacional o local, políticos, líderes religiosos, organizaciones de la sociedad civil y grupos con intereses especiales, los círculos académicos u otras organizaciones. En el mismo orden de ideas Tapella (2007, p. 3) señala que "los actores sociales pueden ser personas, grupos u organizaciones que tienen interés en un proyecto o programa". A su vez la Organización de las Naciones Unidas para la Alimentación y la Agricultura (FAO) observa que "Usualmente son considerados actores aquellos individuos, grupos o instituciones que son afectados o afectan 
el desarrollo de determinadas actividades, aquellos que poseen información, recursos, experiencia, y alguna forma de poder para influenciar la acción de otros" (EC-FAO, 2006). Es decir, a los actores no se les identifica o define en forma independiente del contexto, sino en función de un aspecto específico de la realidad o asunto sobre el cual la gente tiene una acción concreta.

De acuerdo con León (1985), el término organización implica una estructura de funciones o puestos formalizados, por lo que para entenderla es importante conocer los elementos que la conforman. Para el propósito de este estudio se clasifican las organizaciones en tres tipos, de acuerdo con el papel que desempeñan en el manejo de los recursos naturales, es decir, como apropiadores, reguladores y orientadores.

\section{Apropiadores}

En el caso de Marismas Nacionales Sinaloa los apropiadores están representados por las organizaciones de productores. Estas tienden a conformar organizaciones más amplias como federaciones, asociaciones, cooperativas o comités para la extracción, uso o manejo de algún recurso natural. Son entidades económicas orientadas a la producción que se ubican en el sector primario.

Desde este ámbito "desarrollan procesos y formas para la integración del trabajo en estructuras asociativas de carácter empresarial, para el desarrollo cooperativo o colectivo del proceso de producción, de manera total o parcial, para lograr economías de escala que faciliten el acceso a la producción tecnificada y para aumentar los ingresos de los productores" (Dirección General de Organización de los Productores Agrícolas y Forestales [DGOPAF], 1981, p. 92).

Para el propósito de caracterizarlas y poder realizar un análisis más específico, estas organizaciones se dividieron en productores directos e indirectos. Como productores directos se considera a las organizaciones cuyo objetivo es la extracción o el aprovechamiento de los recursos naturales; en MNS existen diversas organizaciones de este tipo enfocadas en agricultura, ganadería, pesca, acuacultura y turismo. Algunos ejemplos de este tipo de organizaciones en la zona de interés son las cooperativas pesqueras y las de prestación de servicios turísticos en pequeña escala.

Por otro lado, se asume que son organizaciones de productores indirectos aquellas cuya función es coordinar los trabajos y efectuar la gestión de los productores directos para el manejo de los recursos naturales. Por ejemplo la Federación de Cooperativas de Producción Pesquera del Sur de Sinaloa y la Asociación Ganadera de Escuinapa. 


\section{Reguladores}

Los organismos reguladores del manejo de los recursos naturales son las dependencias gubernamentales. A estas les corresponde elaborar las directrices y orientaciones para el ordenamiento de recursos naturales específicos o del espacio; establecer lineamientos de uso y ocupación del territorio de acuerdo con los atributos y límites del ambiente, y su correlación con los objetivos de desarrollo.

Este tipo de organizaciones pueden regular los recursos naturales por medio de incentivos y programas sectoriales que apoyan o motivan al productor a desarrollar su actividad. En el caso de México, aplican restricciones como las vedas de los recursos de interés a los usuarios y diversos subsidios que fomentan determinadas prácticas.

\section{Orientadores}

Como orientadores se encuentran las organizaciones de la sociedad civil (OSC). Por OSC se entiende a "organizaciones conformadas por personas que se nuclean en grupos estructurados en base a normas, intereses, objetivos y fines particulares, que tienden a dar respuestas a necesidades sociales, grupales o colectivas" (Acotto, 2003, p. 35). Estas organizaciones pueden aportar a procesos de concertación, información, apoyo técnico, capacitación y ejercer su capacidad de convocatoria. Al respecto la Fundación por la Socialdemocracia de las Américas, A.C. (2009, p. 28), menciona que "Si bien la fundación de estas organizaciones es reciente, en la actualidad éstas se han convertido en actores sociales de gran relevancia en aras del fortalecimiento de una democracia participativa".

Muchas de estas organizaciones tienen objetivos que apuntan a fortalecer el manejo sustentable de los recursos naturales, reorientar los sistemas productivos para mantener y mejorar las condiciones ambientales y que los actores y grupos de interés de las regiones donde intervienen desarrollen capacidades para incidir en la toma de decisiones. En la zona de Marismas Nacionales Sinaloa encontramos diversas organizaciones que orientan el uso o manejo de recursos naturales, que se caracterizan por apoyar a través de asesorías y capacitación a los usuarios.

\section{Contexto socioambiental de Marismas Nacionales Sinaloa}

Como ya se mencionó, Barragán (2004) sostiene que los sistemas socioecológicos se caracterizan por estar integrados por tres subsistemas, el biofísico, el socioeconómi- 
co y el jurídico-administrativo, en este orden de ideas se describe el sistema de Marismas Nacionales Sinaloa.

\section{Subsistema biofísico}

La zona conocida como Marismas Nacionales Sinaloa se localiza en los municipios de Escuinapa y El Rosario, Sinaloa, México; colinda al sur con el estado de Nayarit, en la región conocida como Marismas Nacionales Nayarit. El río de las Cañas al sur y el río Baluarte al norte del sistema son las corrientes más importantes que aportan agua dulce al ecosistema y delimitan la zona, que abarca una superficie de 47,556 hectáreas (Conanp, 2008).

El sistema se conforma por una serie de lagunas y canales. "En su mayor parte son espacios abiertos con vegetación dispersa en sus riberas, siendo el estero El Mezcal de los pocos cuerpos de agua angostos y sinuosos, que se ubican en el área. Las lagunas son de profundidad escasa y muy probablemente sus fondos se eleven por encima del nivel medio del mar" (Meraz Sánchez, 2005, p. 16).

Los ecosistemas de Marismas Nacionales Sinaloa forman una región zoogeográfica en la zona costera, donde se encuentra flora y fauna muy diversa. La flora de la región está conformada por selva baja caducifolia y subcaducifolia y una importante comunidad de manglar que se encuentra en los bordes y zonas adyacentes de esteros y lagunas. La fauna está representada por 380 especies de vertebrados, el grupo de mayor número son las aves playeras y acuáticas, que usan el sitio como estancia invernal y de alimentación; los mamíferos y los peces, con especies de hábitos estuarinos, principalmente, entre las que se encuentran algunas con categoría sujeta a protección especial y de importancia económica, cinegética y alimenticia (Conanp, 2008).

\section{Subsistema socioeconómico}

De acuerdo con información del INEGI (2010), en Marismas Nacionales Sinaloa las actividades económicas se desarrollan principalmente en el sector primario, en el cual se ocupa $55 \%$ de la población; en segundo lugar se encuentra el sector terciario, con aproximadamente $40 \%$, y el tercer lugar lo ocupa el secundario o de transformación, con $5 \%$. La población del municipio de Escuinapa en 2010 fue de 54,131 habitantes, de los cuales 27,301 son hombres y 26,830 mujeres.

En el área se llevan a cabo diversas actividades económicas. Entre las más importantes se encuentran la agricultura de temporal, a la que se destina una superficie de cultivo estimada en 28,295 hectáreas (ha), de las cuales 4,100 están 
ocupadas por cultivos perennes de frutas como mango, ciruela y cocotero, y el resto por cultivos de maíz, frijol y pastos dependientes de las precipitaciones pluviales y de la agricultura de riego, que aprovecha el agua del acuífero de la barra para el riego de hortalizas. Respecto al tipo de tenencia de los terrenos agrícolas, $49.4 \%$ es ejidal y $50.6 \%$ es propiedad privada (Ramírez Zavala et al., 2012).

La ganadería es fundamentalmente extensiva y se basa en la cría de ganado bovino, porcino, caprino y avícola. Predominan el ganado bovino, con cerca de 43,287 cabezas, y el porcino, con cerca de 10,000 cabezas; la producción anual es de 1,154 toneladas del bovino y 335 del porcino. Ocupan el tercer lugar las aves de postura con 5,100 (INEGI, 2010).

La pesca es una de las actividades económicas más representativas de la región y genera una importante cantidad de empleos: 4,500 pescadores están registrados legalmente; la acuacultura se dedica básicamente el cultivo de camarón y tiene un potencial de empleo de 9,100 trabajadores (Ramírez Zavala et al., 2012).

Aunque el turismo es una actividad aún incipiente en la región, esta se encuentra en el circuito turístico Mazatlán-Escuinapa-Teacapán. La infraestructura de servicios para el turismo está integrada primordialmente por pequeños hoteles, restaurantes y cafeterías.

\section{Subsistemajurídico-administrativo}

Encontramos diversas disposiciones de ordenamiento territorial vigentes para la zona de Marismas Nacionales Sinaloa, ya que es considerada por su importancia biológica, ecológica, cultural y recreativa no solo en el ámbito nacional sino también en el internacional, en diferentes categorías de Área Natural Protegida (ANP). Elárea cuenta con un decreto internacional como Sitio Internacional de la Red Hemisférica de Reservas para Aves Playeras (RHRAP), también forma parte del convenio de Humedales Ramsar. Adicionalmente, está considerada en el Plan Estatal de Áreas Naturales Protegidas de Sinaloa.

También se identificaron instrumentos jurídicos, de planeación y regulación del desarrollo en la zona; entre los de mayor importancia están la Ley General de Equilibrio Ecológico y Protección al Ambiente y la Ley General de Vida Silvestre, que regulan el uso de los recursos naturales, así como diversas Normas Oficiales Mexicanas como la NOM-059-SEMARNAT-2010, que regula la protección de especies nativas de México de flora y fauna silvestres con categorías de riesgo, y la Norma Oficial Mexicana NOM-022-SEMARNAT-2003, que establece las especificaciones para la preservación, conservación, aprovechamiento sustentable y restauración de los humedales costeros en zonas de manglar. 
Las actividades económicas que se realizan en la zona están reguladas por las leyes y normas sectoriales y de carácter sanitario. Entre los instrumentos de planeación destacan el Plan Nacional de Desarrollo y los programas sectoriales del Ejecutivo federal, estatal y municipal, así como los Programas Especiales para el Desarrollo Regional Sustentable (Procodes).

\section{FIGURA 1. Ubicación del área de estudio}

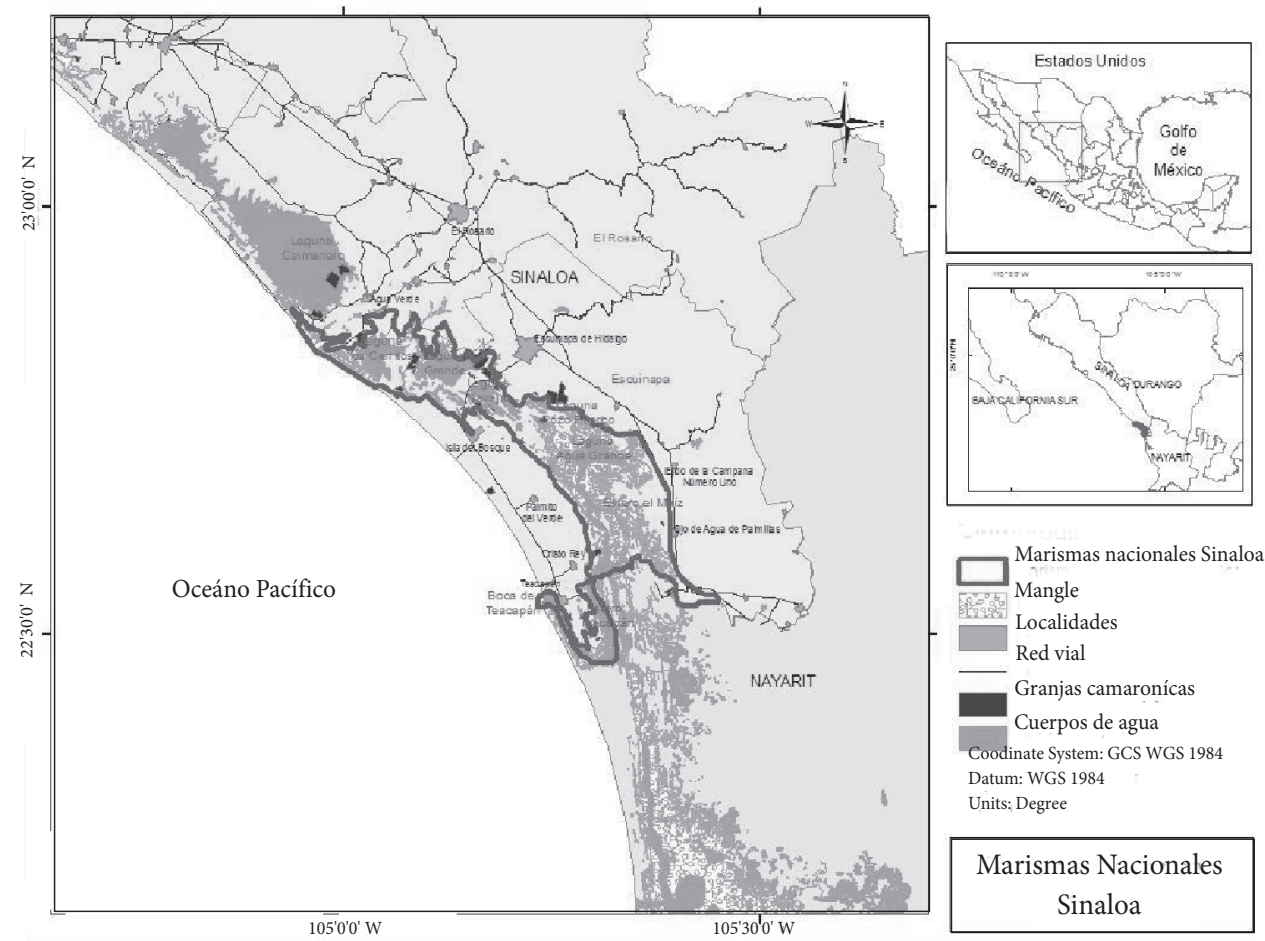

Fuente: Tomado de Bojórquez (2017).

El rol de los actores sociales en la gestión de los recursos de MNS

Organizaciones involucradas en el manejo de los recursos naturales en MNS

Se identificaron 115 actores sociales que pertenecen, representan o declaran a nombre de 99 organizaciones, de las cuales 28 son de apropiadores, es decir, pertenecen al grupo de actores que se dedican a la extracción o producción de 
GRÁFICA 1. Proporción de organizaciones involucradas en el manejo de los recursos naturales en MNS de acuerdo con el papel que desempeñan en él

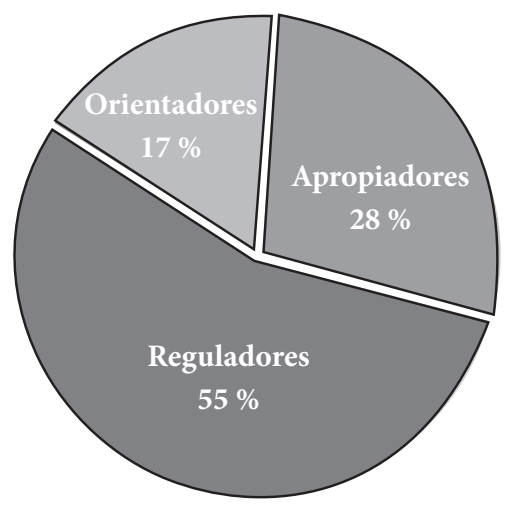

Fuente: Elaboración propia.

recursos naturales y la organización de los mismos, 54 ejercen funciones como reguladores del uso de los recursos naturales y 17 orientan su uso (gráfica 1).

Entre las organizaciones que destacan en el periodo analizado por la frecuencia de sus declaraciones en fuentes hemerográficas están el Centro Integralmente Planeado (CIP) en los diferentes nombres que ha adoptado: Teacapán, Costa Pacífico y Playa Espíritu; el Fondo Nacional de Fomento al Turismo (Fonatur); la Comisión Nacional de Pesca y Acuacultura (Conapesca); la Federación de Cooperativas Pesqueras del Sur de Sinaloa; al menos diez de las cooperativas pesqueras que operan en la zona, y los tres niveles de gobierno: federal, estatal y municipal. Las menciones en medios muestran también que la actividad turística y la pesquera son de gran interés en la zona.

En el análisis de la presencia de las organizaciones de apropiadores en los medios de comunicación impresos se encontró que existe una proporción similar entre la aparición de los apropiadores directos y los indirectos en la prensa escrita (gráfica 2); sin embargo, existe una concentración importante de registros para la Federación de Cooperativas Pesqueras del Sur de Sinaloa entre los apropiadores indirectos, y destaca la influencia de dicha organización en la pesca de la región, mientras que entre los apropiadores directos la mayor cantidad de registros se distribuye entre cuatro cooperativas pesqueras, lo que resalta la influencia de la actividad pesquera en la región. 


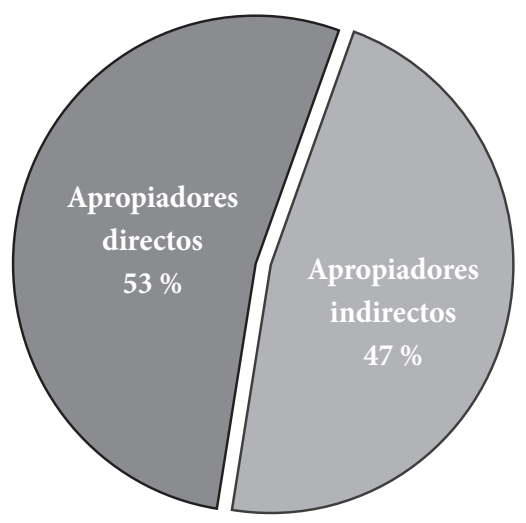

Fuente: Elaboración propia.

Con respecto a las organizaciones reguladoras del uso de los recursos naturales en el área de estudio, fueron divididas en las que basan su funcionamiento en restricciones utilizando el marco normativo y aquellas que basan su funcionamiento

GRÁFICA 3. Proporción de organizaciones de reguladores que utilizan restricciones, incentivos o ambos recursos para realizar su labor

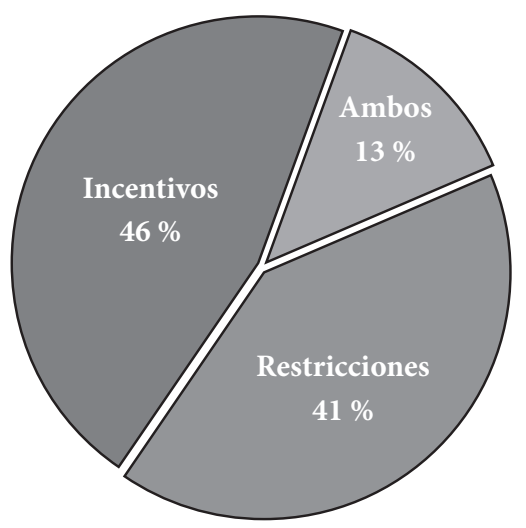

Fuente: Elaboración propia. 
en incentivos, y otros organismos que se valen de ambas formas de regulación. Se advierte una proporción similar de organismos que hacen uso de incentivos y restricciones como forma de operar, $46 \%$ y $41 \%$ respectivamente, mientras que el 13 $\%$ tiene atributos y facultades para utilizar ambas formas (gráfica 3 ).

Con respecto a la escala espacial en que operan las organizaciones identificadas, encontramos que $45 \%$ opera a escala local, $36 \%$ a escala nacional, $13 \%$ a escala regional y solo $6 \%$ tiene influencia internacional (gráfica 4). Porcentajes relacionados muy probablemente con la jurisdicción federal que prevalece en la mayoría de los recursos naturales de importancia en la zona de estudio y, por supuesto, con las actividades de las organizaciones locales. Entre las que destacan por su activa participación están Conapesca, el CIP, la Federación de Cooperativas Pesqueras del Sur de Sinaloa, Fonatur y los gobiernos municipal, estatal y federal.

GRÁFICA 4. Escala espacial en que operan las organizaciones involucradas en el manejo de recursos naturales en MNS

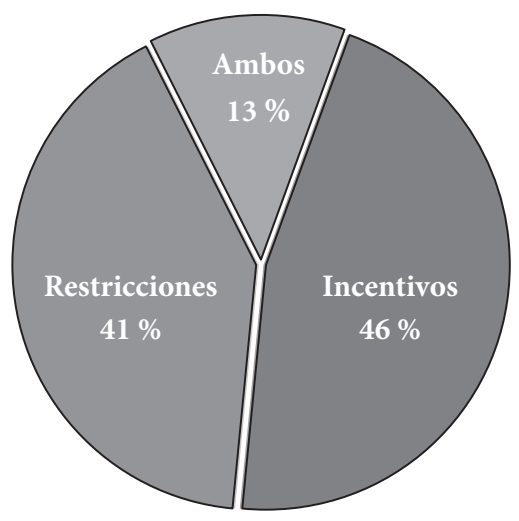

Fuente: Elaboración propia.

Recursos naturales de importancia social en MNS

Con respecto a los recursos naturales sujetos a algún esquema de manejo o que generan conflictos que se reflejan en los medios masivos de comunicación impresos y electrónicos, se generaron 181 registros. Destacan el camarón (86 registros) y los esteros y marismas como ecosistemas (37 registros)entre los recursos que generan mayor preocupación en la sociedad en general y entre los involucrados en el manejo de los recursos naturales de MNS (gráfica 5). 


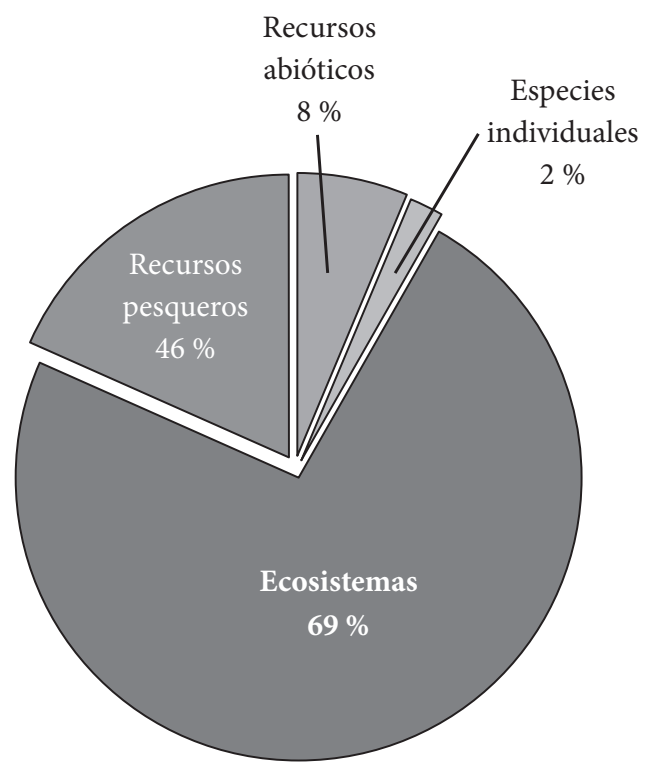

Fuente: Elaboración propia.

\section{Caracterización de los actores}

La caracterización de los actores se realizó siguiendo el enfoque de gobernanza ambiental, a partir del cual se dividieron los actores en apropiadores, reguladores y orientadores, de acuerdo con su papel en el manejo. Los datos que arroja la encuesta permiten un primer acercamiento al tipo de organización, sus intereses, formas de ocupación del territorio y las relaciones que mantienen con otras organizaciones que intervienen en el manejo de los recursos naturales en la región.

\section{Caracterización de las organizaciones de apropiadores}

De las organizaciones encuestadas resalta que las principales actividades de los apropiadores directos son extractivas y de producción primaria como la pesquera, agrícola y ganadera. Mientras que los apropiadores indirectos concentran sus actividades en coordinar los trabajos de las organizaciones de productores y la gestoría para el acceso a programas de apoyo de sus agremiados. 
Las zonas o áreas donde llevan a cabo sus actividades están restringidas a los límites del municipio de Escuinapa, particularmente en las zonas de lagunas y esteros, en tierras de vocación agrícola y ganadera de propiedad ejidal y de pequeños propietarios del municipio. En el caso de las organizaciones dedicadas a las actividades agrícolas y ganaderas, así como a las turísticas, se detectó que operan durante todo el año; en el de las dedicadas a la pesca, por el carácter estacional de su actividad, concentran sus operaciones de manera temporal en una parte del año. Además, mientras que las temporadas de pesca tienden a reducirse, las agrícolas, al secuenciar diferentes tipos de cultivos, ofrecen ocupación durante la mayor parte del año; igual que las actividades turísticas, que tratan de mantenerse activas también durante todo el año, aunque tienen temporadas altas y bajas por la afluencia estacional del turismo en la región.

Con respecto a la tecnología utilizada para el aprovechamiento de los recursos naturales, la pesca incluye diversos métodos y artes de pesca, que abarcan tapos (arte de pesca fijo), atarrayas, chinchorros, redes y trampas, que se han venido usando desde hace décadas. En el caso de la agricultura los métodos, técnicas y especies cultivables se han incrementado en años recientes debido al aumento del nivel de tecnificación de esta actividad en la región, y lo mismo ocurre con la ganadería, mientras que el turismo realiza solo operaciones en pequeña escala. La mayor parte de la producción local se comercializa en los mercados local y nacional.

Mientras que en la pesca y la ganadería disminuye constantemente la producción, la agricultura y el turismo muestran una tendencia positiva; se observan aumentos importantes en la producción de hortalizas, en particular de algunas variedades de chiles, así como mejoras en la afluencia turística. En este sentido, el descenso en el sector de la pesca en la producción se atribuye al azolve y la degradación del ecosistema debido a su envejecimiento natural, acelerado por procesos erosivos derivados de actividades antropogénicas, la contaminación y malas prácticas de pesca, que repercuten negativamente en la condición de los recursos naturales en que se sustenta esta actividad. Los ganaderos, por su parte, atribuyen el declive de su actividad a la falta de rentabilidad.

Los agricultores consideran que los aumentos en la producción se deben a las mejoras tecnológicas que han adoptado, mientras que las eventuales disminuciones que observan en algunos años se deben principalmente a factores climáticos. La actividad turística ha mejorado en la medida en que ha mejorado la infraestructura necesaria para la prestación de este tipo de servicios y la promoción del destino.

Todas las actividades productivas de la región reciben diversos tipos de apoyos, económicos y en especie, en diferentes montos y con distinta frecuencia. Por ejemplo, la pesca recibe fondos gubernamentales principalmente para el funcionamiento de programas como el subsidio de gasolina, sustitución de motores fuera de 
borda, apoyo para la reparación de infraestructura, empleo temporal, desazolve de marismas y esteros y Propesca, que es un apoyo a los pescadores para su manutención en época de veda. La agricultura recibe este tipo de apoyos para la adquisición de maquinaria e infraestructura; apoyo en casos de desastres naturales como falta de floración (en cultivos perennes), inundaciones; asesoría técnica y económica para la exportación. Por su parte, el turismo ha recibido apoyos para infraestructura, promoción y capacitación tanto de agencias gubernamentales como de organismos de la sociedad civil.

Entre los organismos que proporcionan estos apoyos mencionaron a dependencias del gobierno federal como Comisión Nacional de Acuacultura y Pesca (Conapesca), Secretaría de Agricultura, Ganadería, Desarrollo Rural, Pesca y Alimentación (Sagarpa), Conanp, a un diputado federal, al Gobierno del Estado de Sinaloa, organismos mixtos del sector, la Confederación de Cooperativas Pesqueras, la Unión Ganadera Regional de Escuinapa, la Confederación de Asociaciones de agricultores y organismos de la sociedad civil como SUMAR.

Los entrevistados declaran que la mayoría de los programas se orientan a entregar apoyos directamente a la persona beneficiada, aunque algunos se canalizan por medio de las organizaciones gremiales, como el subsidio a la gasolina que se otorga a las cooperativas y los insumos agrícolas que se canalizan a través de las asociaciones de agricultores.

Todas las actividades identificadas están reguladas, por lo que el acceso a los recursos o el desarrollo de la actividad requiere la acreditación de derechos, que los usuarios respaldan a través de permisos y concesiones, en el caso de la pesca y el turismo, y de escritura pública para la agricultura y la ganadería.

Entre las principales dependencias a donde acuden a realizar los trámites de derechos de uso mencionaron Conapesca, Sagarpa, Secretaría de Desarrollo Agrario, Territorial y Urbano (Sedatu) y Secretaría de Economía para las actividades pesqueras, agrícolas y ganaderas, y Capitanía de Puerto, Secretaría del Medio Ambiente y Recursos Naturales (Semarnat) y Conanp para el turismo. Las mismas que regulan el acceso y control de los recursos se encargan de su aplicación, y todas las actividades, excepto el turismo, participan de alguna manera en la vigilancia de las prácticas de sus agremiados y otros usuarios legales e ilegales de los recursos.

Aunque es activa su participación en estas labores, para lo cual se coordinan en la realización de estos operativos con las mismas dependencias y con miembros de cuerpos de seguridad como la Marina, el Ejército, policías estatales y municipales, declaran que las organizaciones con las que establecen mejores mecanismos de colaboración son las integradoras de la actividad y las agencias gubernamentales del sector, por lo que muchos recursos económicos y humanos se atomizan y pierden así efectividad. 
Las actividades que realizan las dependencias que atienden el sector de la pesca se refieren a gestión de trámites, otorgamiento de permisos de nuevo ingreso, renovación, entrega de avisos de arribo, cosecha, producción, guías de pesca y administración de las granjas acuícolas y cooperativas. Las dependencias relacionadas con la agricultura gestionan apoyos a productores, ejidatarios, personas que se dedican a la ganadería o la agricultura, a quienes asesoran para gestionar recursos para proyectos específicos; un ejemplo de esto son los apoyos para la construcción de represas para el aprovechamiento del agua.

El carácter de área natural protegida de la región requiere de la presencia de dependencias que promueven actividades para la conservación del medio ambiente, por ejemplo los programas de reforestación de mangle; promocionar y difundir las que realizan generalmente organizaciones de productores, clubes u organizaciones de la sociedad civil. Sin embargo, las oficinas locales de las dependencias gubernamentales identificadas generalmente no cuentan con programas de apoyo o subsidios a productores con recursos y reglas de operación propios, solo canalizan los trámites a niveles superiores de la dependencia y organizan la entrega de apoyos que asignan las oficinas centrales.

Los principales programas de financiamiento, equipamiento, apoyo, subsidio, capacitación y asesoría técnica a productores que aterrizan en la región son Procampo, apoyo para energía eléctrica, gasolina ribereña, diésel marino, y Propesca y otros apoyos económicos. En funciones de gestoría, las oficinas locales sirven de enlace para apoyar a las cooperativas en buscar en recursos por medio de proyectos con base en reglas operativas de dependencias federales y del gobierno del estatal para capacitación a productores, artesanos y prestadores de servicios turísticos.

Además en la parte ambiental, particularmente Conanp, promueve el Programa de Conservación para el Desarrollo Sostenible (Procodes), que también apoya proyectos de conservación en cursos de capacitación, estudios técnicos y proyectos. Otras actividades que realizan tienen relación con campañas de recolección de envases de agroquímicos, para que no se depositen en basureros clandestinos ni generen contaminación.

Estos programas están orientados a ejidatarios, pescadores, productores independientes, prestadores de servicios de los sectores turístico y empresarial. Los apoyos derivados de estos programas se otorgan directamente a personas físicas o morales o a través de las organizaciones gremiales.

Los objetivos del o los apoyos, según las dependencias consultadas, son fomentar la producción agrícola, aumentar y mejorar la producción, fortaleces la 
economía de los productores y sus capacidades, así como el desarrollo de actividades productivas diversas para la conservación de los recursos.

Las principales leyes o normas que regulan estas actividades relacionadas con los recursos son: Ley General de Desarrollo Social, Ley de Desarrollo Rural Sustentable y Ley General de Pesca y Acuacultura Sustentable, y sus respectivos reglamentos; Normas Oficiales Mexicanas, así como las reglas de operación de los programas de apoyo por sector productivo; Ley General de Ecología y Protección del Ambiente, Ley General de Vida Silvestre, reglamentos y normas del sector ambiental y Ley de Metrología y Normalización.

Las oficinas locales de estas dependencias no son competentes para llevar a cabo acciones o programas de regulación, monitoreo, inspección y vigilancia; aunque sirven de enlace, coordinación y apoyo a las dependencias encargadas de estas tareas, en su mayoría no apoyan a otras dependencias en estas labores, por lo que su participación es mínima en este sentido. Por otro lado, las dependencias que sí tienen atribuciones para el monitoreo y la vigilancia realizan estas actividades en coordinación con otras dependencias gubernamentales y las organizaciones de productores.

La mayoría de estas organizaciones federales y estatales no aplican ningún tipo de sanción, se limitan a utilizar procedimientos administrativos y canalizar infractores a instancias del poder judicial para complementar trámites relacionados con el uso indebido de recursos naturales de jurisdicción federal. La autoridad local tiene atribuciones para amonestar, multar, cancelación temporal o definitiva de algunos tipos de permiso; concesión, licencia o autorización de jurisdicción municipal; la clausura parcial o total, temporal o definitiva de establecimientos que violen la normatividad local, y el arresto de personas físicas.

Los recursos regulados por las dependencias mencionadas son recursos pesqueros, agua, mangle, suelo, esteros y marismas, recursos forestales. Algunas dependencias solo regulan la actividad. El presupuesto propio que ejercen es muy limitado, en su mayoría solo administran recursos federales que ejercen para gasto corriente, programas de apoyo, cursos, estudios y proyectos.

\section{Caracterización de las organizaciones de orientadores}

Estas organizaciones tienen una presencia importante en la región; realizan actividades de fortalecimiento de las capacidades de la sociedad para generar liderazgos enfocados en el aprovechamiento sustentable de los recursos naturales. Otorgan apoyo a productores y reguladores para capacitación y concientización sobre el aprovechamiento de los recursos naturales, entre ellos a la Conanp y cooperativas de ecoturismo y pesca. 
Para realizar estas actividades se coordinan con algunas dependencias gubernamentales, organizaciones de productores u otras organizaciones de la sociedad civil como Conselva, SUMAR voces por la naturaleza y Centro Mexicano de Derecho Ambiental (CEMDA). Realizan sus actividades con fundamento en la Ley de Fomento a las Actividades Realizadas por Organizaciones de la Sociedad Civil. Por eso no se les permite involucrarse en acciones de regulación, monitoreo, inspección y vigilancia, pero sí apoyan organizaciones de productores y dependencias del gobierno en labores de inspección y vigilancia, como la ayuda que prestan a pescadores. Enfocan sus esfuerzos en la conservación del paisaje, los recursos pesqueros y el fomento de buenas prácticas agrícolas, pesqueras, acuícolas y turísticas; su presupuesto está compuesto fundamentalmente de donativos.

\section{Conclusiones}

El análisis de la presencia de los actores sociales en los medios de comunicación muestra la influencia en la región de la actividad pesquera. Aunque ya no representa un porcentaje importante del PIB regional como hace tres décadas, la problemática ambiental y social que se deriva del deterioro de recursos pesqueros, ecosistemas que los sustentan y usuarios de la pesca, tienen un impacto importante en la vida cotidiana de las comunidades y un efecto mediático significativo por el interés que suscita el tema en la sociedad local y regional.

Las organizaciones identificadas llevan a cabo actividades de aprovechamiento en los sectores pesquero, agrícola, ganadero y turístico, principalmente. Existen actividades no registradas en el periodo de estudio, como la acuícola y minera, que aunque es de suponer que tienen importancia económica, ambiental y productiva posiblemente mayor que otras actividades que sít tuvieron presencia en los medios de comunicación impresa, sus registros hemerográficos son escasos; fue prácticamente nula sobre todo la presencia de la minería, pues la acuacultura es una actividad que, por llevarse a cabo en espacios que colindan e incluso se traslapan con las actividades pesqueras, agrícolas, ganaderas y turísticas, es más visible, aunque su problemática no se ventile en los medios de comunicación.

Entre las limitaciones del estudio se encuentran sus reducidas posibilidades para trazar una tipología precisa de los actores sociales, pues la base de datos se limita a la consulta hemerográfica, por lo que la minería y la acuacultura tienen un perfil bajo en los medios y no se han podido registrar aun cuando la experiencia de campo prueba que son actividades importantes. Por ello se recomienda que en estudios posteriores se amplíe la base de datos en cuanto al tiempo, el número de medios consultados y las fuentes de información. 
En la problemática acerca de los recursos naturales (181 registros totales) destacan las declaraciones sobre el camarón como recurso ( 86 registros) y los esteros y marismas como ecosistemas (37 registros), cuya degradación causa una gran preocupación a los habitantes y a quienes estén involucrados en el manejo de los recursos naturales de MNS por la influencia cultural de la pesca de camarón en la región y la dependencia que guardan muchas personas con los bienes y servicios que generan los ecosistemas.

De las principales actividades registradas, la pesca y la ganadería han tenido una disminución en su producción, mientras que la agricultura y el turismo muestran una tendencia positiva, fenómeno que el sector pesca atribuye principalmente a la degradación del ecosistema, que repercute negativamente en los recursos naturales que sustentan su actividad. Los agricultores, como sector de mayor crecimiento económico en el periodo de estudio, observan que las mejoras tecnológicas que han adoptado los han beneficiado; sin embargo, perciben como amenaza las anomalías climáticas que afectan los cultivos.

Aunque las organizaciones de la sociedad civil tienen una presencia importante en la región porque realizan actividades enfocadas en el aprovechamiento sustentable de los recursos naturales, es necesario establecer puentes de comunicación entre las organizaciones de los diferentes sectores que permitan la construcción de políticas integrales que aborden de mejor manera los problemas de manejo que se observan en la región, a la vez que optimizan recursos materiales y humanos, ya que es evidente que las organizaciones que operan en la región tienen mecanismos de colaboración con organizaciones propias de cada sector en función de programas de apoyo de diferente tipo, desarticulados entre sí, que los organismos gubernamentales y civiles proporcionan a los productores.

Esta sectorización de los esfuerzos atomiza los recursos humanos y financieros e impide construir políticas públicas que impulsen medidas que aborden la problemática de manera integral. La región adolece de organismos intersectoriales que promuevan la cooperación entre los diversos actores con diferentes intereses pero con problemas comunes, lo cual socava las posibilidades de un desarrollo que incorpore criterios de sustentabilidad.

Tampoco existen regulaciones o instrumentos de ordenación que permitan organizar las actividades que utilizan los recursos naturales que funcionen de manera efectiva. Aunque se identificaron instrumentos de manejo, como las diferentes denominaciones de ANP, que proponen regular el espacio, las actividades y los recursos presentes en la zona, su implementación está en manos de una dependencia con escaso poder operativo, político y económico que es la Conanp, lo que limita las posibilidades para su aplicación y de conservación efectiva de la base de recursos que dé soporte al desarrollo sustentable de las actividades económicas y 
contribuya a mejorar la calidad de vida de los habitantes asentados en el área de influencia de Marismas Nacionales Sinaloa.

\section{Referencias}

Acotto, L. (2003). Las organizaciones de la sociedad civil. Un camino para la construcción de ciudadanía. Buenos Aires: Espacio Editorial.

Banco Mundial (2007). Relación con la comunidad y otros actores sociales. Manual de prácticas recomendadas para las empresas que hacen negocios en mercados emergentes. Washington, D.C.: Corporación Financiera Internacional. Recuperado de www.ifc.org/enviro [consultado el 14 enero de 2017].

Barragán Muñoz, J. M. (2003). Medio ambiente y desarrollo en áreas litorales. Introducción a la planificación y gestión integradas. Cádiz: Servicio de Publicaciones Universidad de Cádiz.

Barragán Muñoz, J. M. (2004). Las áreas litorales de España. Del análisis geográfico a la gestión integrada. España: Editorial Ariel.

Bojórquez Sauceda, J. (2007). La gobernanza de recursos de uso común: la pesca de camarón en Marismas Nacionales Sinaloa. Tesis doctoral, Doctorado en Ciencias Sociales, El Colegio Mexiquense, A.C. Toluca de Lerdo, Estado de México.

Comisión Nacional del Agua (Conagua) (2007). Guía para la identificación de actores claves. México:Semarnat.Recuperado de http://www.conagua.gob.mx/CONAGUA07/

Noticias/IAC.pdf [consultado el 21 de octubre del 2016].

European Commission-Food and Agriculture Organization of the United Nations (EC-FAO) (2006). Stakeholders analysis. Annex I to lesson "understanding the users' information needs", Food Security Information for Action Programme, EC-FAO-EU.

Farhad, S. (2012). Los sistemas socio-ecológicos. Una aproximación conceptual y metodológica. En XIII Jornadas de Economía Crítica: Departamento de Economía, Métodos Cuantitativos e Historia Económica (pp. 265-280). España: Universidad Pablo de Olavide.

Fundación por la Socialdemocracia de las Américas, A.C. (2009). Nueva visión socialdemócrata. Sociedad en movimiento. Revista, 16, 23-26. Recuperado de http://www.fusda. org/Revista16/Revista16-[consultado el 25 de enero de 2017].

Grimble, R. \& Wellard, K. (1996, abril). Stakeholder methodologies in natural resource management: a review of principles, contexts, experiences and opportunities. Documento presentado en el ODA NRSP Socioeconomic Methodologies Workshop, Londres, 29-30.

Tapella, E. (2007). El mapeo de actores claves. Documento de trabajo para el proyecto 
Instituto de Investigación de Recursos Biológicos Alexander von Humboldt, Centro de Cooperación Indígena (2005). Caracterización de actores ambientales del Piedemonte Andino-Amazónico. Colombia: IAVH-CECOÍN.

Instituto Nacional de Estadística y Geografía (INEGI) (2010). Anuario estadístico y geográfico de Sinaloa 2010. Aguascalientes: INEGI.

Instituto Nacional de Estadística y Geografía (INEGI) (2014). Anuario estadístico y geográfico de Sinaloa 2014. Aguascalientes: INEGI.

León Betancourt, A. (1985). Organizaciones y administración: un enfoque de sistemas. Cali: Norma.

Meraz Sánchez, R. (2005). Evaluación de la condición del bosque de manglar utilizando datos provenientes de sensores remotos y su relación con la producción pesquera, en el sistema lagunar Chametla-Teacapán, Sinaloa, México. Tesis de licenciatura, Facultad de Ciencias del Mar, Universidad Autónoma de Sinaloa. Culiacán, Sinaloa.

Ostrom, E. (2007). A diagnostic approach for going beyond panaceas. Proceedings of the National Academy of Sciences, 104(39), 15181-15187.

Programa de las Naciones Unidas para el Medio Ambiente (PNUMA) (2009). Gobernanza ambiental. Nairobi, Kenya: PNUMA, División de Derecho Ambiental e Instrumentos Internacionales.

Ramírez Zavala, J. R., Cervantes Escobar, A. \& Ramírez Zavala, J. R. (2012). El ambiente biofísico de Marismas Nacionales, Sinaloa, y criterios básicos para la gestión de su integridad ecológica. En Ramírez Zavala, J. R., Cervantes Escobar, A. \& Tapia Hernández, F. J. (coords.), Marismas Nacionales Sinaloa: futuro y conservación. Culiacán: Pronatura Noroeste, A.C., Universidad Autónoma de Sinaloa.

Ramírez Zavala, J. R., Ramírez Zavala, J. R., Bojórquez Sauceda, J.Santos Guzmán, S. \& Herrera y Cairo Lizárraga, M. C. (2012). El socio-ecosistema Marismas Nacionales Sinaloa frente al futuro. ¿Sustentabilidad o colapso? En Ramírez Zavala, J. R., Cervantes Escobar, A. \& y Tapia Hernández, F. J. (coords.), Marismas Nacionales Sinaloa: futuro y conservación. Culiacán: Pronatura Noroeste, A.C., Universidad Autónoma de Sinaloa.

Resilience Alliance (2010). Assessing resilience in social-ecological systems: Workbook forpractitioners. Version 2.0. Recuperado de https://www.researchagate.net/publication /269576211Assessingresilienceinsocialecological_systems_Workbook_for_practitione rs_Version_20 [consultado el 27 de junio de 2017].

Sánchez Anaya, A. (1981). Cap. V. Organización de productores. En: Seminario de evaluación de la ley de fomento agropecuario. México: Instituto de Investigaciones Jurídicas/Facultad de Medicina Veterinaria y Zootecnia, : UNAM.

Secretaría de Agricultura, Ganadería, Desarrollo Rural, Pesca y Alimentación (Sagarpa) (2009). Las organizaciones económicas del sector rural. Principios y bases jurídicas. Boletín. 
Recuperado de http://www.sagarpa.gob.mx/desarrolloRural/AsistenciaCapacitacion /Documents/Centros\%20de\%20Evaluacion/utes/desemp/formacion/Boletin_ORGANIZ _ECON_BASES_JURIDICAS_2009.pdf [consultado el 23 de septiembre de 2016].

Tapella, E. (2007). El mapeo de actores claves. Documento de trabajo para el proyecto "Efectos de la biodiversidad funcional sobre procesos ecosistémicos, servicios ecosistémicos y sustentabilidad en las Américas: un abordaje interdisciplinario". Universidad Nacional de Córdoba, Inter-American Institute for Global Change Research.

Tréllez, E. (2000). La educación ambiental y las utopías del siglo XXI. Tópicos en Educación Ambiental, 2(4). 